\title{
دراسة كفاءة الأداء للبناء الجاهز دراسة تأثير ليزر النيديميوم- زجاج على خواص العزل الكهريائي للمواد السيراميكية
}

\author{
م.م. نادية محمد جاسم \\ قسم الفيزياء- كلية العلوم- جامعة ديالى ماديه

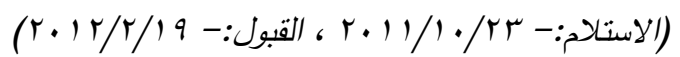

الخلاصة

( ليزر النيدميوم- زجاج ، السيراميك، ثابت العزل الكهربائي) ان الهدف من إجراء هذا البحث هو دراسة تأثثر ليزر النيديميوم-زجاج على خواص العزل الكهربائي للجسم السيراميكي ومقارنة نتائج ثابت العزل الكهربائي للجسم السيراميكي بدون نأثير أنتعة الليزر • ان طريقة العمل تضدنت جزئين : الجزء الأول تم حساب ثابت العزل الكهربائي لجسم سيراميكي محضر من ثلاث مواد وهي( كاؤولين دويخلة، رمل زجاج ارضمة وفلدسبار البوتاسيوم) وبسمك (3-3.5mm) باستخدام جهاز LCR meter من نوع Agilenty 4294Aparcil Imperlence حيث تم حساب قيمة ثنابت العزل الكهربائي للجسم السيراميكي Dielectrically Constant من قيمة المتسعة الناتجة من تغير التردد. إما الجزء الثاني من البحث فقد تضمن تسليط اثتعة ليزر النيديميوم زجاج وبطاقات مختلفة E= 600, 700, 800mJ، على النماذج السيراميكية وحساب ثابت العزل الكهربائي لها مرة أخرى ومن ثم مقارنة قيمة ثابت العزل الكهربائي للنماذج السيراميكية معهد وبدون تأثنير أشععة الليزر .

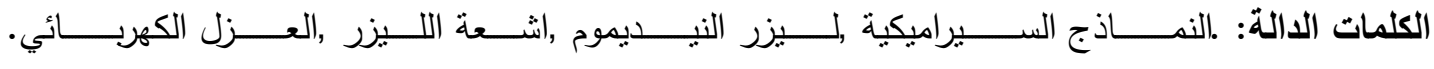

تثقيز معظم المواد السيراميكية بالمقاومة الكهربائية النوعية العالية،وذلك بسبب الترابط بين ذرات المواد التي تكون

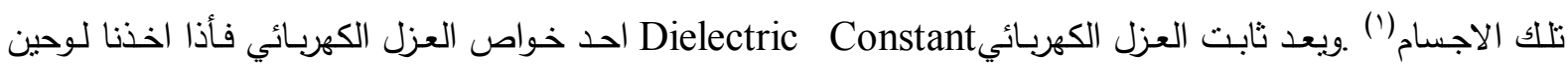
معدنيين متوازيين مساحة كل منهما (A) فأنهما يكونان متسعة سعتها (Co)وتعتمد على سماحية الفراغ 60 او سماحية

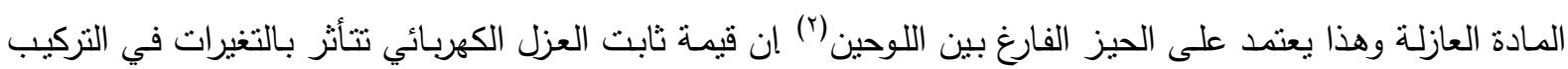

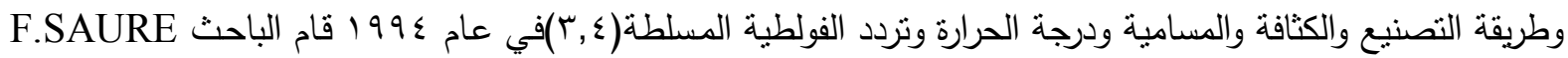

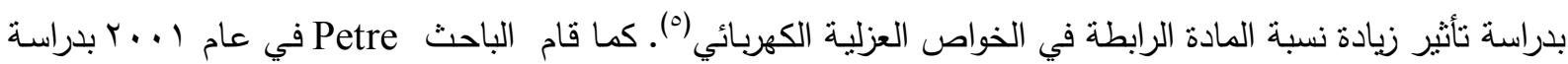
تأثنر المواد الملانة والمواد الرابطة والمواد المزيتة على خواص المواد السيراميكية حيث انه يمكن تتفيذ نظام القولبة بالحقن

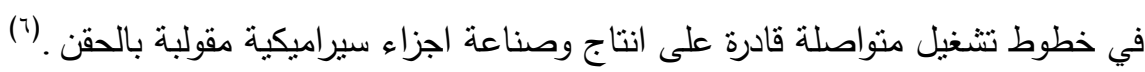

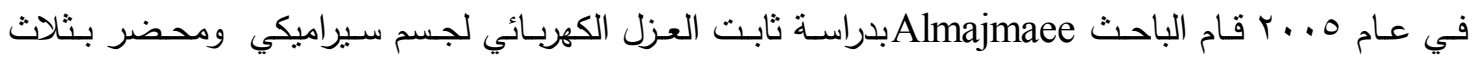
خلاطات (حيث كل خلطة تحتوب على نسبة وزنية معينة من المادة الرابطة Binder وكانت Polyvinybutyral ومادة 


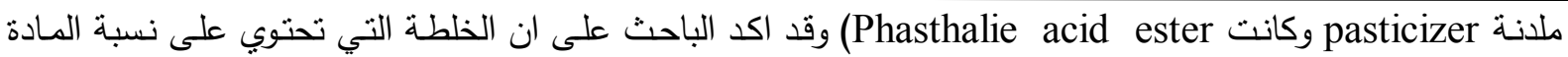
الرابطة اكثر هي التي اعطت اعلى ثابت عزل (v) ملانه

\section{الجزء العملي}

ا-حساب ثابت العزل الكهربائي للجسم السيراميكي بدون تأثير اثعة الليزر تضمن هذا الجزء حساب قيمة ثابت العزل

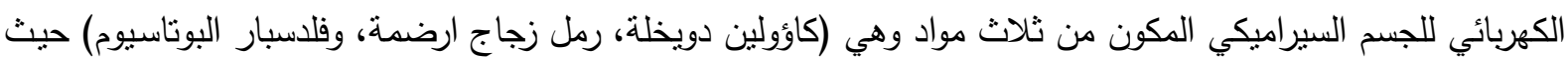

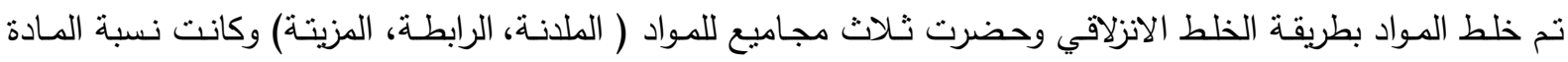

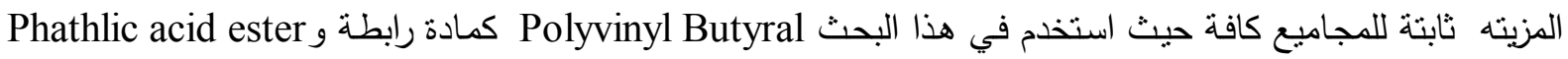

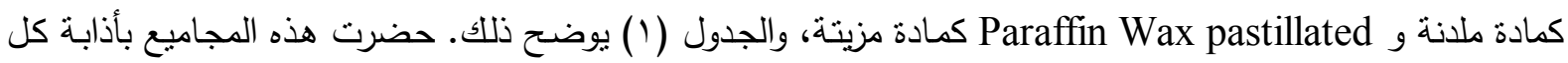

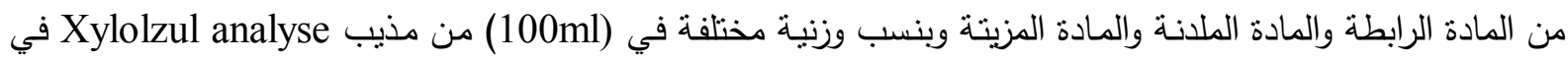

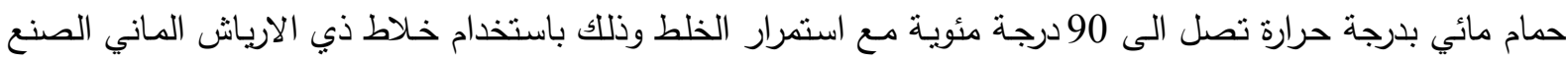

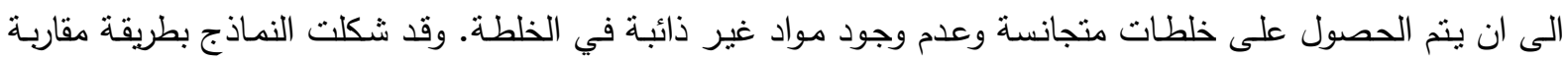

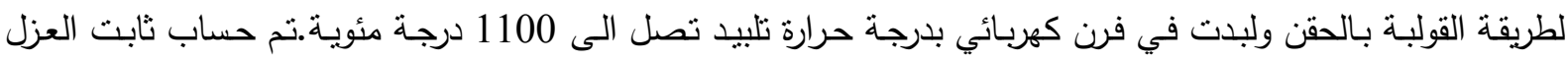

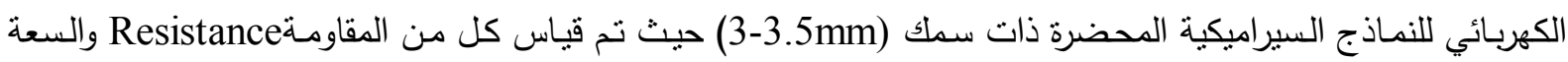
Capacity وباستخدام جهاز LCR meter وبتسليط فولنيـة مقدارها 500mV وبذلك تم حساب ثابت العزل الكهربائي Dielectric Constant

$$
C o=\frac{\varepsilon_{\circ} A}{d}
$$

وتدعى (ع) بسماحية الفراغ وهي كمية ثابتة وتساوي (10,9]

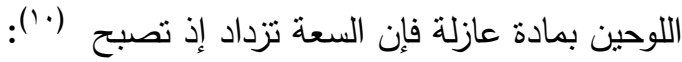

$$
\begin{aligned}
C & =\frac{\varepsilon A}{d} \\
\varepsilon & =\frac{C d}{A}
\end{aligned}
$$

$$
\begin{aligned}
& \text { وتدعى (ع) بسماحية المادة العازلة التي تفصل اللوحين أو السماحية المطلقة ولها نفس وحدات (F/m)()). } \\
& \text { ومن المعادلتين(r) و (r)يمكن ملاحظة أن نسبة قيمة السعة عند وجود العازل هي (·(') : } \\
& \frac{C}{C_{O}}=\frac{\varepsilon}{\varepsilon_{O}}
\end{aligned}
$$

وتدعى هذه النسبة بالسماحية النسبية (Relative Permittivity) أو ثابت العزل (Dielectric Constant) ويرمز لـه بالرمز 'ع والتي يمكن تعريفها بأنها النسبة بين سماحية المادة إلى سماحية الفراغ، أب أن:

$$
\begin{gathered}
\varepsilon^{\prime}=\frac{\varepsilon}{\varepsilon_{O}} \\
\varepsilon^{\prime}=\left(\frac{1}{\varepsilon_{o}}\right)\left[\frac{d}{A} C\right]
\end{gathered}
$$$$
\text { بتعويض المعادلة(ع ) و (0) نحصل على (9): }
$$ 
ثانيا-حساب ثابت العزل الكهربائي للجسم السيراميكي بتأثنر اشعة ليزر النيديميوم زجاج في الجزء الثاني من البحث تم

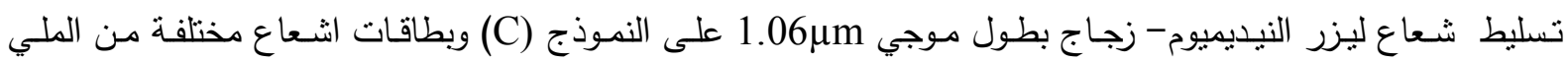

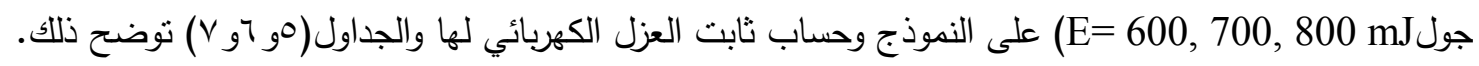

\section{مناقشة النتائج}

- حساب ثابت العزل الكهربائي للجسم السيراميكي بدون تأثير اثعة الليزر . من خلال النظر الى الجداول(والاشكال

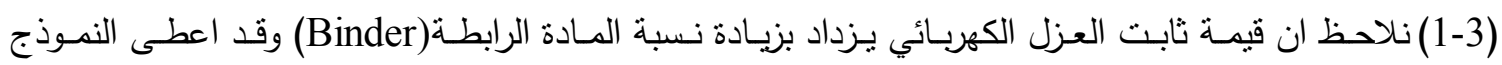

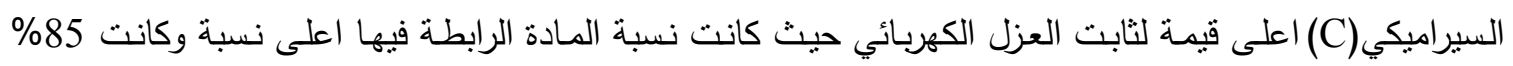

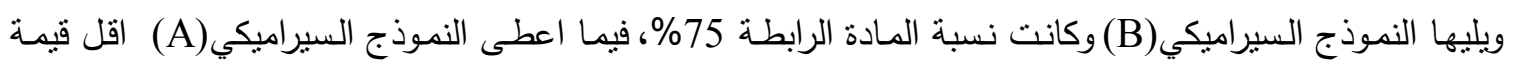

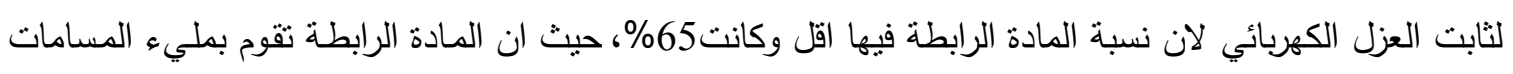
الموجودة في التركيب البلوري مما يؤدي الى نقصان المسامية التي تساهم في عملية التوصيل الكهربائي وبالتالي لتئي سوف تؤدي الىى زيادة الكثافة وهذا بدوره يؤدي الى تحسين الخواص العزلية الكهربائية للنموذج السيراميكي، وقد فئد

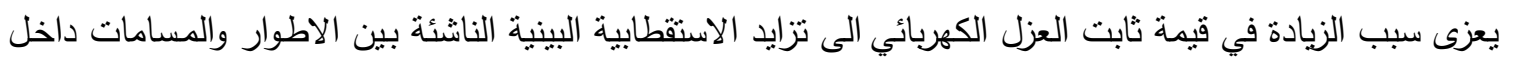

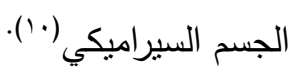

r- حساب قيمـة ثابت العزل الكهربائي للجسم السيراميكي بتأثير اشعة ليزر النيديميوم-زجاج. تم اختيار النموذج

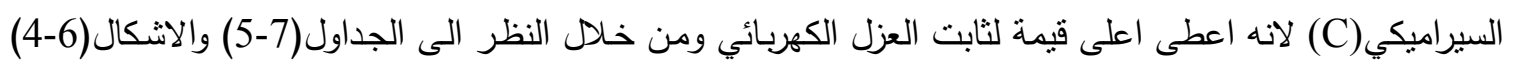
نلاحظ ان قيمة ثابت العزل الكهربائي بزداد بزيادة الطاقة الليزرية المسلطة عليه وليه وهذا بدوره يؤدي الى تقليل مسامية

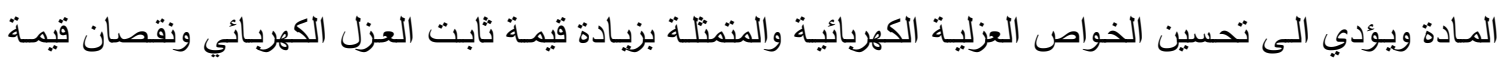

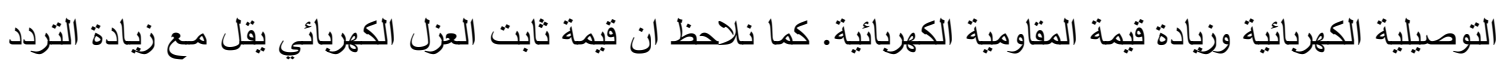

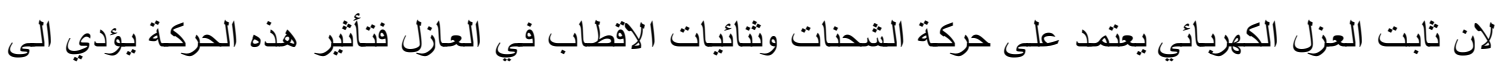

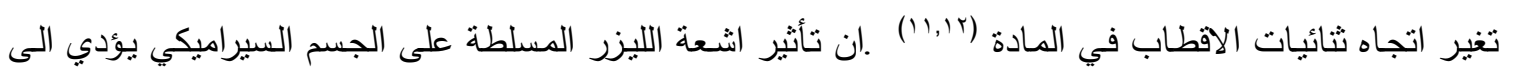
الحصول على جسم صلب ومتماسك متحولا بذلك الجسم السيراميكي من جسم مسامي الى جسم قوي وكثيف وذلك

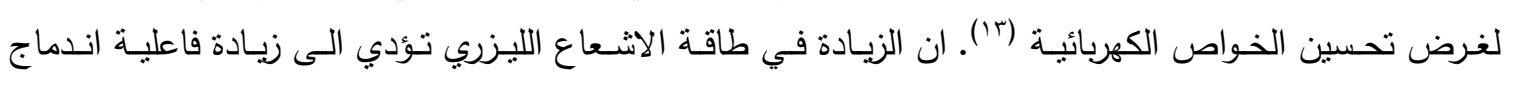

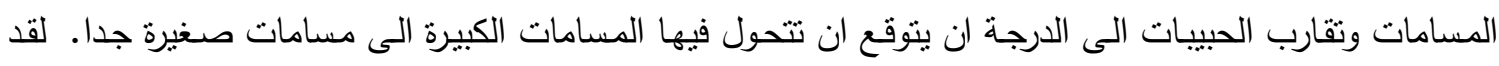

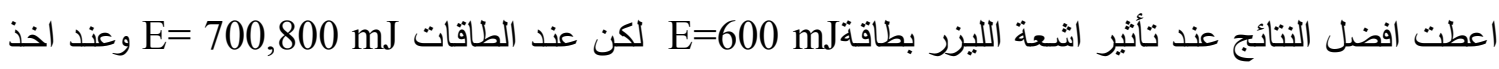
القيم لثاب العزل الكهربائي نلاحظ حصول الخطأ عند قراءة الجهاز ذلك لحصول التشققات في الجسم السيراميكي حيث ادى التقلص عالي جدا وظهور التشققات لذلك يجب ان تكون الطاقة الليزريـة المسلطة اقل من هذه القيم لتجنب انكماش الجسم السيراميكي الى درجة كبيرة وظهور التشققات وهذا بدوره لايؤثر فقط على الخواص الكهربائية للجسم السيراميكي بل يؤثز ايضا على الخواص الميكانيكية ومنها متانة الجسم السيراميكي. 
ا-كلمـا زادت نسبة المـادة الرابطـة في المـادة السيراميكية (Binder) كلما تحسنت الخـواص العزليـة الكهربائية وقلت

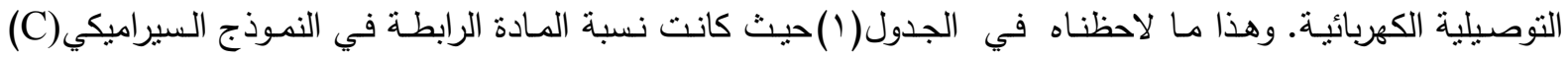
85\% وهي اعلى نسبة وقد اعطت اعلى قيمة لنابت العزل الكهربائي وكان 6.05 ץ- ان تأثير اشعة ليزر النيديميوم-زجاج على الجسم السيراميكي يؤدي الى تحسين خواص العزل الكهربائي حيث يزداد

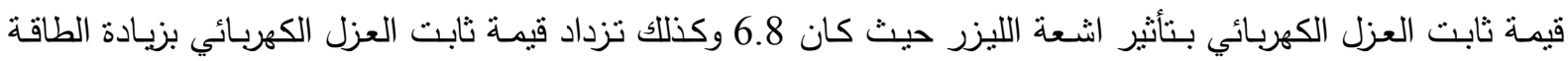
الليزرية لكن يجب الانتباه أن لا نؤدي زيادة الطاقة إلى حدوث نشققات في الجسم السيراميكي وهذا ماحصل عند تسليط انشعة الليزر بطاقات Mj ب- ان وجود الفراغـات (المسامات) في الجسم السيراميكي وبإحجـام كبيـرة يؤدي إلـى نقصان في قيمـة ثابـت العزل الكهربائي وزيادة التوصيلية الكهربائية وهذا بدوره يؤثر سلبيا على الخواص العزلية الكهربائية.

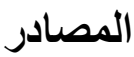

ا. شـروق صـباح عبد العباس، دراسـة الخـواص الفيزيائيـة للعـازل الكهربـئي السيراميكي ذي الجهـد العـالي"، رسـالة

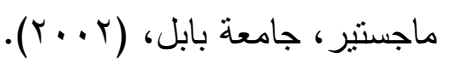

2-W. D. Kingery, (1960) "Thermal Conductivity of Ceramic Dielectric Progress in Ceramic Science", Vol. 2, J. E. Burke ed. Pergamon on Press).

3-Blumethal, R. N. and Setiz. M. A., (1989) "Experimental Techniques Electrical Conductivity in Ceramic and Glass, Part (A), (N. M.Tallened), Mrcel Dekker Inc., New York.

4-Tareev, B., (1979) "Physics of Dielectric materials", Mir Publishers, Moscow, pp. 13 - 16, $44-48$.

5- F. Saure., (1994), shwand of Federal republic of Germany" Injection moulding" Inter ceramic, vol.33, pp.[1-2].

6- Peter R,(2001) ," An approach to cost Efficient preparation of ceramic injection moulding compounds", Inter ceramic, vol.23, No.1.

7- Al- Majmaee, A,M, 2005 "The Use of Plastic Component Pyramid for Forming a

Ceramic body and Studying some physical properties",thesis,Msc, Al- Mustansiria University.

8-Harrop, P. J., (1972) "Dielectrics Butter Worth and Co., Publishers, Ltd. London, Butter Worths.

9- www.ceramic materials (2011).

10- Omar, M. A., (1975) "Elementary Solid State physics: Principles and Applications: Addition Wesley Publishing Company Inc., London.

11-Smyth, Ch. Ph., (1955) "Dielectric Behavior and Structure", McGraw - Hill Book Company Inc., New York. 
دراسة كفاءة الأداء للبناء الجاهز دراسة تأثير ليزر النيديميوم- زجاج على خواص العزل الكهريائي للمواد السبراميكية 12-Kinser, D. L., (1971) "Electrical Conduction in Glass and Glass Ceramics. Physics of Electronic Ceramics, Part A, (L. L. hench and D. B. Dove ed.), Marcel Dekker, Inc., New York.

13-Gone folgen, (2005) " Laser material processing" Hand Book, New York.

$$
\text { جدول( () يوضح نسب مكونات النماذج السيراميكية( الملدنة، الرابطة، المزيتة) والمواد الداخلة في تكوين النموذج }
$$

\begin{tabular}{|c|c|c|c|c|c|c|c|}
\hline النموذج رمز & $\begin{array}{c}\text { الرابطة Binder } \\
\text { المادة }\end{array}$ & $\begin{array}{l}\text { المادة الملدنة } \\
\text { Pasticizer }\end{array}$ & المادة المزيتة & $\begin{array}{c}\text { المذادة } \\
\text { Solvent } \\
\text { المذيبة }\end{array}$ & كاؤولين & زرجاج & فلبونار البونيوم \\
\hline & $\begin{array}{l}\text { Polyvinyl } \\
\text { butyral }\end{array}$ & $\begin{array}{l}\text { Phathalie } \\
\text { acidester }\end{array}$ & $\begin{array}{l}\text { Paraffin } \\
\text { pastillated }\end{array}$ & $\begin{array}{c}\text { Xylolzul } \\
\text { Analyse }\end{array}$ & النوزنية\% & الوزنبة\% & الوزنية\% \\
\hline & النسبة الوزنية\% & النسبة|لوزنية\% & النسبة الوزنية\%\% & النسبة ml & & & \\
\hline A & 65 & 25 & 10 & 100 & 20 & 25 & 30 \\
\hline B & 75 & 15 & 10 & 100 & 30 & 25 & 30 \\
\hline $\mathrm{C}$ & 85 & 5 & 10 & 100 & 40 & 25 & 30 \\
\hline
\end{tabular}

\begin{tabular}{|c|c|c|}
\hline $\begin{array}{l}\text { Dielectric constant } \\
\varepsilon^{\prime}\end{array}$ & $\mathrm{Rp}$ & $\begin{array}{l}\text { Frequency } \\
\mathrm{F}\end{array}$ \\
\hline 4.47 & $1.9^{*} 10^{7}$ & 40 \\
\hline 4.37 & $2.13 * 10^{6}$ & $7.5 * 10^{4}$ \\
\hline 4.3 & $8.65 * 10^{5}$ & $1.5 * 10^{5}$ \\
\hline 4.23 & $4.98 * 10^{5}$ & $2.26 * 10^{5}$ \\
\hline 4.18 & $3.76^{*} 10^{5}$ & $3 * 10^{5}$ \\
\hline 4.05 & $3.3 * 10^{5}$ & $3.82 * 10^{5}$ \\
\hline
\end{tabular}

جدول(ץ): يوضح مقدار ثابت العزل الكهربائي للنموذج السيراميكي(A). 
دراسة كفاءة الأداء للبناء الجاهز دراسة تأثير ليزر النيديميوم - زجاج على خواص العزل الكهريائي للمواد السيراميكية

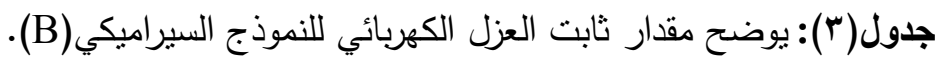

\begin{tabular}{|c|c|c|}
\hline $\begin{array}{l}\text { Dielectric constant } \\
\varepsilon^{\prime}\end{array}$ & $\mathrm{Rp}$ & $\begin{array}{l}\text { Frequency } \\
\quad \mathrm{F}\end{array}$ \\
\hline 5.62 & $2.47 * 10^{5}$ & $3.75 * 10^{5}$ \\
\hline 5.58 & $1.86 * 10^{5}$ & $4.5^{*} 10^{5}$ \\
\hline 5.41 & $1.47 * 10^{5}$ & $5.25 * 10^{5}$ \\
\hline 5.32 & $1.22 * 10^{5}$ & $6.00 * 10^{5}$ \\
\hline 5.22 & $1.00 * 10^{5}$ & $6.78^{*} 10^{5}$ \\
\hline 5.03 & $9.97 * 10^{4}$ & $6.91 * 10^{5}$ \\
\hline
\end{tabular}

جدول(§): يوضح مقدار ثنابت العزل الكهربائي للنموذج السيراميكي(C).

\begin{tabular}{|c|c|c|}
\hline $\begin{array}{c}\text { Dielectric constant } \\
\varepsilon^{\prime}\end{array}$ & $\mathrm{Rp}$ & $\begin{array}{l}\text { Frequency } \\
\text { F }\end{array}$ \\
\hline 6.05 & $8.59 * 10^{4}$ & $7.5^{*} 10^{5}$ \\
\hline 5.88 & $7.67 * 10^{4}$ & $8.25 * 10^{5}$ \\
\hline 5.78 & $6.57 * 10^{4}$ & $9.00 * 10^{5}$ \\
\hline 5.60 & $5.86^{*} 10^{4}$ & $9.76 * 10^{5}$ \\
\hline 5.51 & $5.53 * 10^{4}$ & $1.05 * 10^{6}$ \\
\hline 5.33 & $5.18 * 10^{4}$ & $1.14 * 10^{6}$ \\
\hline
\end{tabular}


جدول(ه): يوضح قيمة ثابت العزل الكهربائي للنموذج السيراميكي (C) عند تسليط اشعة ليزر النيديميوم- زجاج E=600mJ بطاقة

\begin{tabular}{|c|c|c|}
\hline $\begin{array}{c}\text { Dielectric constant } \\
\varepsilon^{\prime}\end{array}$ & $\mathrm{Rp}$ & $\begin{array}{c}\text { Frequency } \\
\mathrm{F}\end{array}$ \\
\hline 6.82 & $8.90 * 10^{3}$ & $3.40^{*} 10^{6}$ \\
\hline 6.50 & $8.22 * 10^{3}$ & $3.55^{*} 10^{6}$ \\
\hline 6.42 & $7.71 * 10^{3}$ & $3.80 * 10^{6}$ \\
\hline 6.30 & $7.10^{*} 10^{3}$ & $3.90 * 10^{6}$ \\
\hline 6.18 & $6.89 * 10^{3}$ & $4.00 * 10^{6}$ \\
\hline 5.91 & $6.50 * 10^{3}$ & $4.20^{*} 10^{6}$ \\
\hline
\end{tabular}

جدول(†): يوضح قيمة ثابت العزل الكهربائي للنموذج السيراميكي (C) عند نسليط اشعة ليزر النيديميوم-زجاج بطاقة E=700mJ.

\begin{tabular}{|c|c|c|}
\hline $\begin{array}{c}\text { Dielectric constant } \\
\varepsilon^{\prime}\end{array}$ & $\mathrm{Rp}$ & $\begin{array}{l}\text { Frequency } \\
\mathrm{F}\end{array}$ \\
\hline 6.63 & $8.85 * 10^{4}$ & $7.80 * 10^{5}$ \\
\hline 6.30 & $7.98 * 10^{4}$ & $8.11^{*} 10^{5}$ \\
\hline 6.23 & $7.36^{*} 10^{4}$ & $8.30 * 10^{5}$ \\
\hline 6.07 & $7.22 * 10^{4}$ & $8.98 * 10^{5}$ \\
\hline 5.73 & $6.43 * 10^{4}$ & $4.59 * 10^{6}$ \\
\hline 4.30 & $8.65 * 10^{5}$ & $9.03 * 10^{6}$ \\
\hline
\end{tabular}


جدول(V): يوضح قيمة ثابت العزل الكهربائي للنموذج السيراميكي(C) عند تسليط اشعة ليزر النيديميوم-زجاج E=800mJ بطاقة E

\begin{tabular}{|c|c|c|}
\hline $\begin{array}{l}\text { Dielectric constant } \\
\varepsilon^{\prime}\end{array}$ & $\mathrm{Rp}$ & $\begin{array}{l}\text { Frequency } \\
\mathrm{F}\end{array}$ \\
\hline 6.76 & $8.89^{*} 10^{4}$ & $7.9^{*} 10^{5}$ \\
\hline 6.16 & $7.82 * 10^{4}$ & $8.34 * 10^{5}$ \\
\hline 4.40 & $2.13^{*} 10^{6}$ & $7.5^{*} 10^{4}$ \\
\hline 6.03 & $8.59 * 10^{4}$ & $7.3^{*} 10^{5}$ \\
\hline 4.18 & $3.3^{*} 10^{5}$ & $3.0^{*} 10^{5}$ \\
\hline 5.75 & $6.23^{*} 10^{4}$ & $9.0^{*} 10^{5}$ \\
\hline
\end{tabular}

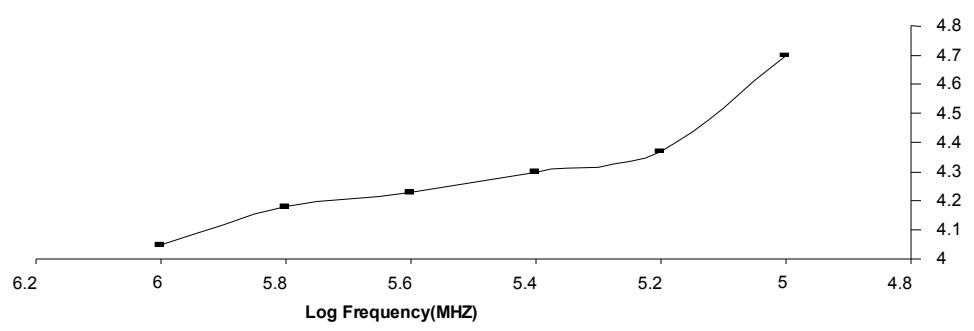

شكل ( ): يوضح مقدار التغير الحاصل في مقدار قيمة ثابت العزل الكهربائي للنموذج السيراميكي

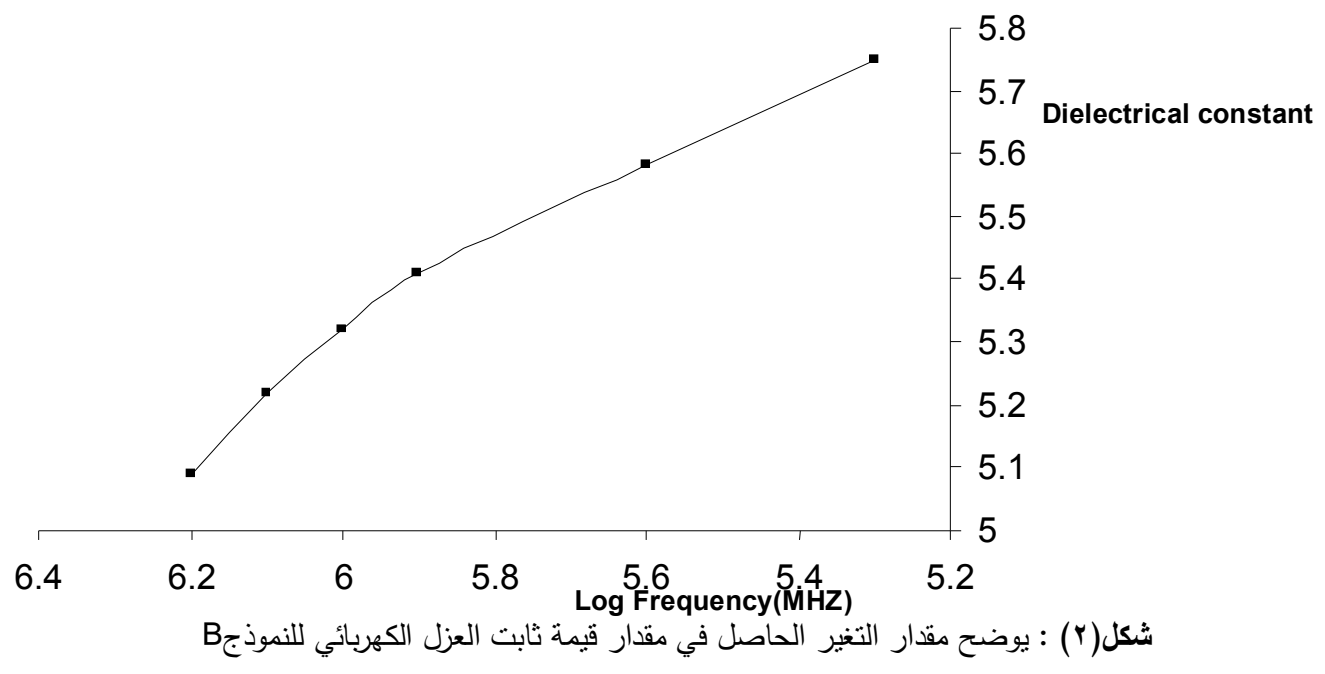




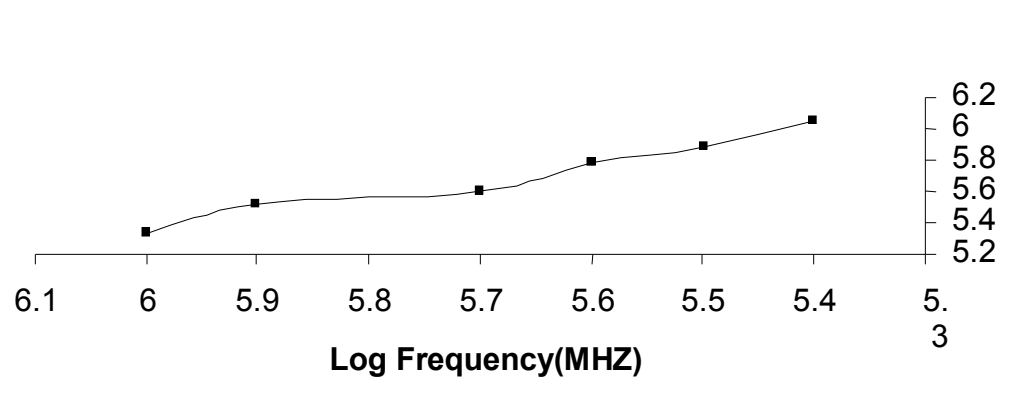

Dielectrical constant

Log Frequency(MHZ)

شكل(r) : يوضح مقار التغير الحاصل في مقدار قيمة ثابت العزل الكهربائي للنموذج

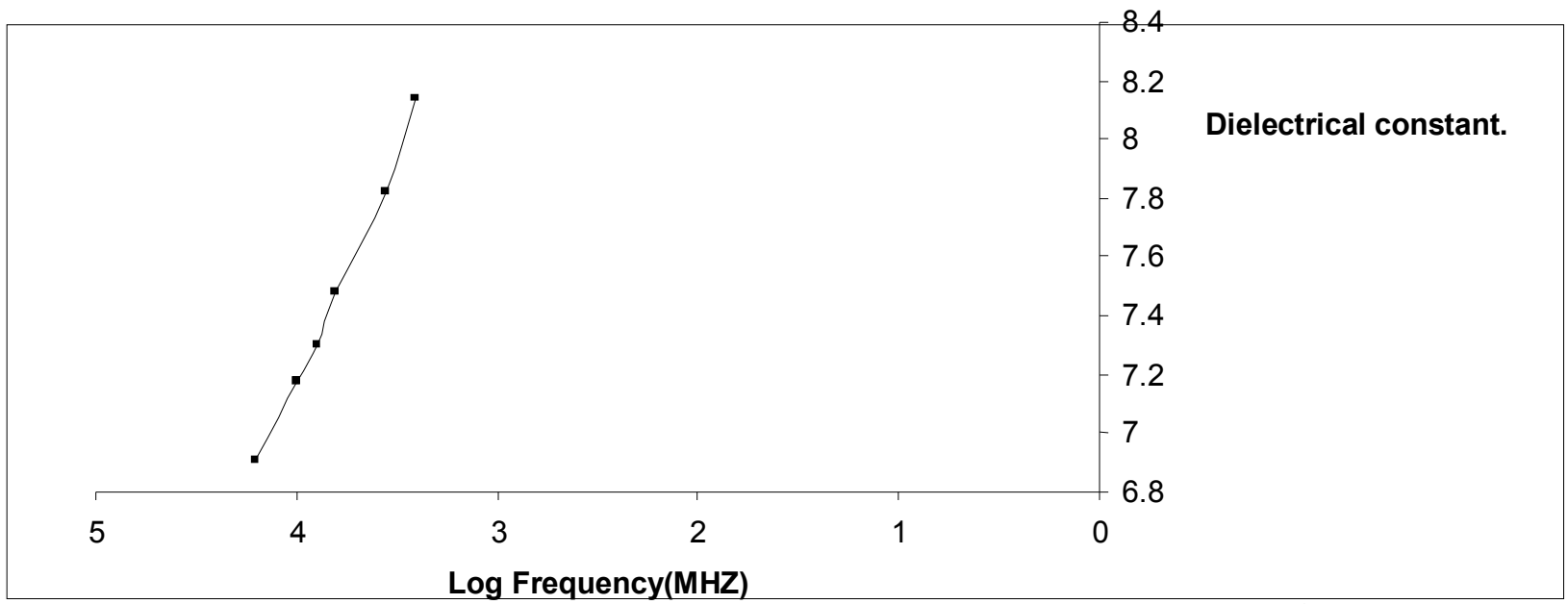

شكل(§): يوضح تأثير اشعة لبزر النيديميوم زجاج على مقدار التغير الحاصل في مقدار قيمة ثابت العزل الكهربائي للنموذجC بطاقة

$E=600 \mathrm{~mJ}$

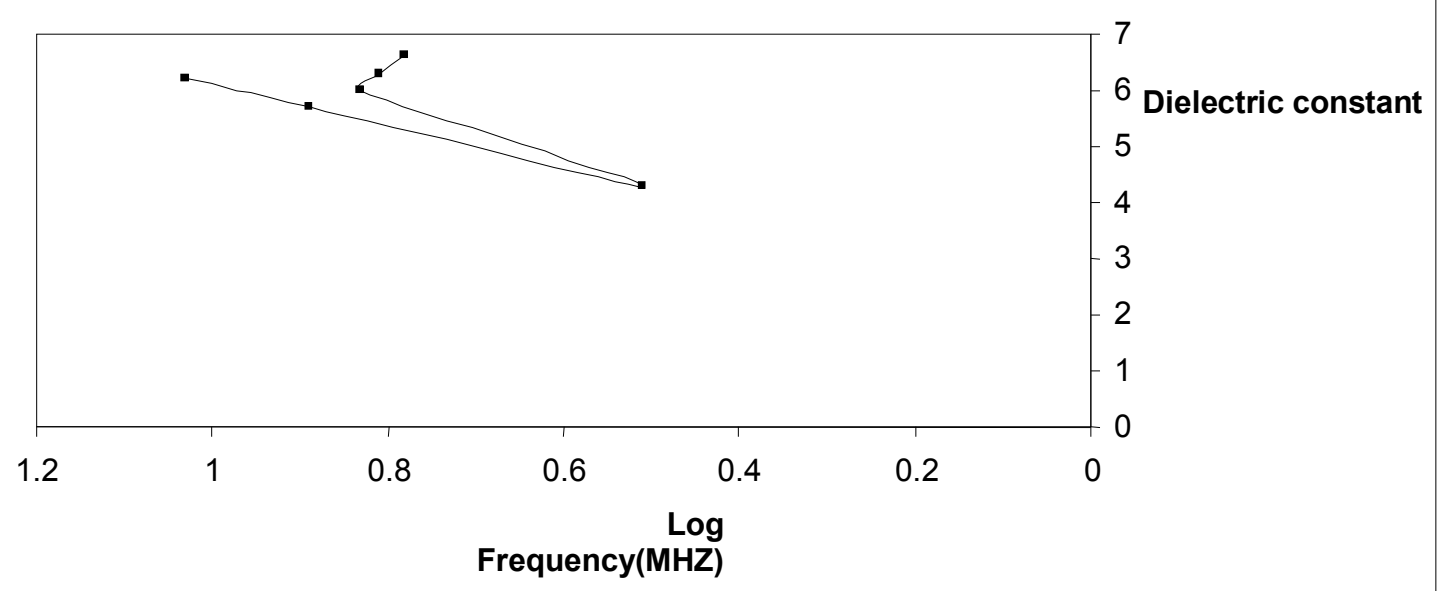

شكل(0): يوضح تأثير اشعة ليزر النيديميوم زجاج على مقدار التغير الحاصل في مقدار قيمة ثابت العزل الكهربائي

E = للنموذجC بطاقة 


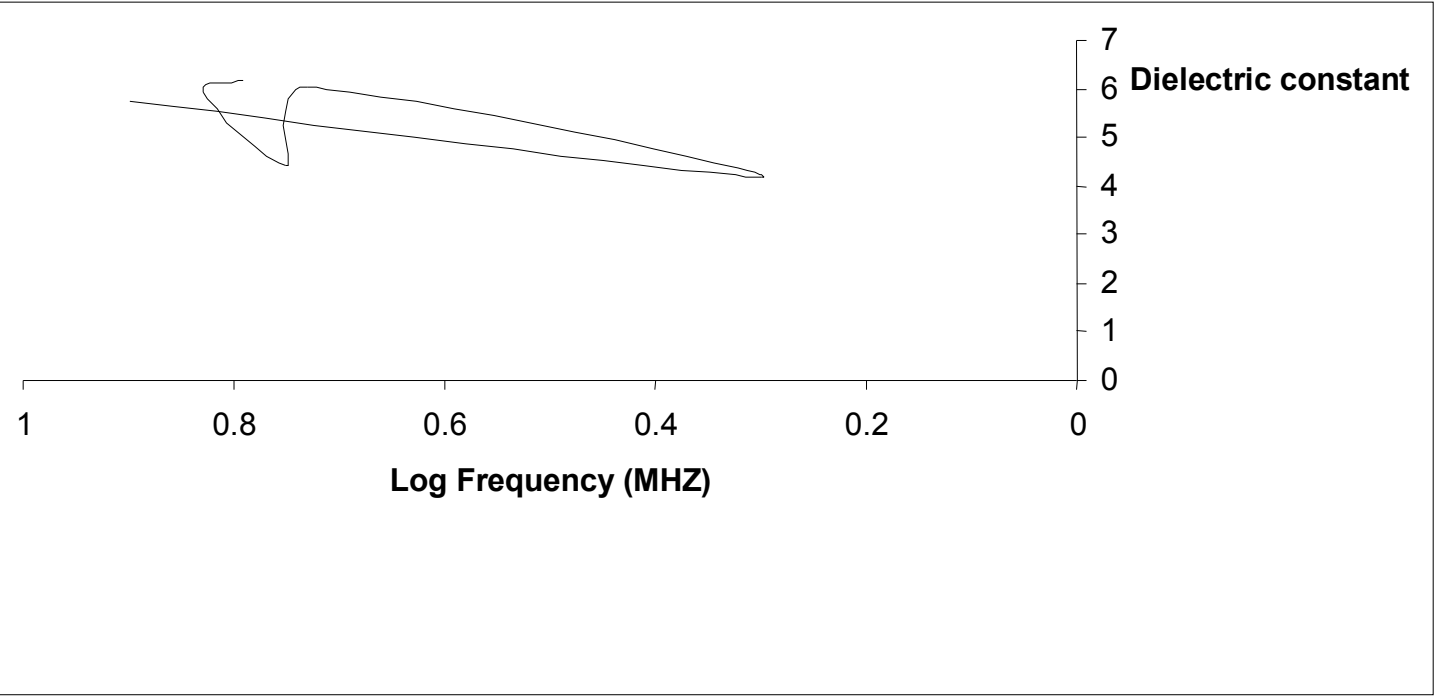

شكل(ף): :يوضح تأثثر اشعة ليزر النيديميوم زجاج على مقدار التغير الحاصل في مقدار قيمة ثابت العزل الكهربائي

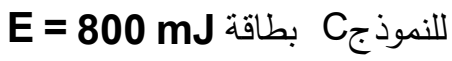


دراسة كفاءة الأداء للبناء الجاهز دراسة تأثير ليزر النيديميوم - زجاج على خواص العزل الكهريائي للمواد السيراميكية

\title{
STUDYING THE EFFECT OF ND-GLASS LASER ON THE DIELECTRICALLY PROPERTIES OF CERAMIC MATERIALS
}

\author{
Nadia Mohammed Jassim(Assist Instructor) \\ Department of physics, College of Science, Diyala University
}

\begin{abstract}
Nd}$ - Glass laser. Ceramic, dielectrically constant) The aim of the present research is to study the effect of Nd-glass laser to the dielectrically properties of the ceramic body and compare the results with and without the effect of laser. The experimental method includes two part: part one includes the calculation of the dielectrically constant of the ceramic body that's formed from three materials(kaolin Dukala,Quartz, potassium fildspar ) with thickness 3-3.5mm by using LCR meter, type Agilenty 4294Aparcil Imperlence. So we calculation the value of the dielectrically constant from the value of the produced amplitude from the changeable frequency. Part two from the research includes the effect of Nd-glass laser radiation with different energies $\mathrm{E}=600,700,800 \mathrm{Mj}$, to the ceramic sample and calculation the dielectrically constant too, and then compare this value for the ceramic samples with and without the effect of laser radiation.
\end{abstract}

